NASA/TM- - E /- 207535

$$
\begin{aligned}
& \text { Pe, W W } \\
& \text { UN \% } \\
& 07960
\end{aligned}
$$

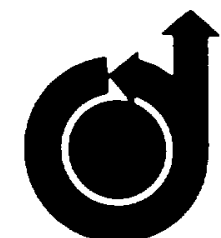

AIAA 80-0117R

Entry and Landing Probe for Titan

J.P. Murphy, J.N. Cuzzi, A.J. Butts

and P.C. Carroll

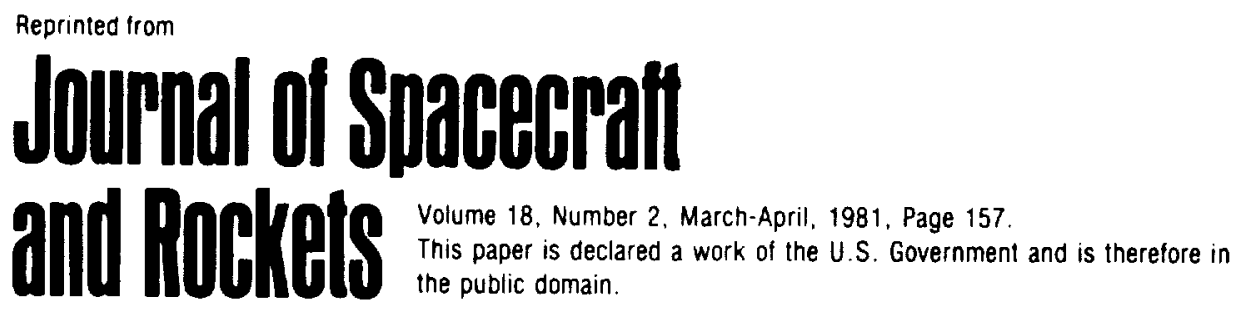




\title{
Entry and Landing Probe for Titan
}

\author{
J.P. Murphy* and J.N. Cuzzi† \\ NASA Ames Research Center, Moffett Field, Calif. \\ and \\ A.J. Butts $\ddagger$ and P.C. Carroll§ \\ Martin Marietta Aerospace Co., Denver, Colo.
}

\begin{abstract}
Results of a recent study of entry and landing probes for the exploration of Titan are presented. The probes considered were based on a wide range of exploration mission possibilities. They included: an atmospheric science probe, an intermediate atmospheric and limited surface science probe and a larger atmospheric and expanded surface science probe. Because of lower gravity on Titan and its atmospheric characteristics, the entry environment is less severe than that of Mars. However, the large uncertainties in the current definition of the atmosphere and the uncertainties in Titan's surface characteristics have required tradeoffs of various combinations of entry and descent shapes and hard lander configurations. Results show that all probe classes are feasible without major developments.
\end{abstract}

\section{Introduction}

$\mathbf{T}$ HE growing interest in Saturn and its largest moon, Titan, has resulted in the definition of exploratory science objectives for Titan from the 1975 National Academy of Science/Space Science Board Report on Space Science and from the 1978 Reston Workshop on the Saturn System. From the Workshop, two extreme atmospheric models were proposed as being the best candidates for describing the actual range of atmospheric parameters possible on Titan. These models ${ }^{1}$ provide the basis for probe system design in this study. In addition, both vehicle and mission design studies of Saturn orbiter and probe systems have been conducted in the period 1975-1978. ${ }^{2.3}$ Results of those studies provided a starting point for this new evaluation.

This paper describes the results of an evaluation of entry and landing probes for the exploration of Titan. The purpose of the study was to define technical requirements, conceptual designs, and science data return of probes designed to accommodate the large atmospheric and surface uncertainties of Titan. The intermediate class lander probe is emphasized. The intermediate lander provides pre-entry, atmospheric, and short-duration surface science. By comparison, the simple atmospheric probe could not meet the requirements associated with the surface science, and the large, expanded lander probe concept exceeded the allowable mass for the baseline Saturn orbiter mission. Therefore, these extreme designs are discussed no further here, although they do provide important system tradeoff data in the study of Ref. 4 .

The intermediate probe design described here meets the basic science requirements for pre-entry and atmospheric and surface science, consistent with the results of the NAS/Space Science Board and Reston Workshop recommendations. The probe system mass is compatible with the Saturn orbiter mission defined in Ref. 3. No major new developments are required to implement this basic Titan probe design.

\section{Mission Overview}

The Titan probe mission would be launched in conjunction with the Saturn orbiter and Saturn probe (SOP). ${ }^{2}$ The Shuttle

Presented as Paper 80-0117 at the AlAA 18th Aerospace Sciences Meeting, Pasadena, Calif., Jan. 14-16, 1980; submitted Jan. 18, 1980; revision received Sept. 29, 1980. This paper is declared a work of the U.S. Government and therefore is in the public domain.

-Assistant Chief, Project Technology Branch.

†Research Scientist.

$\ddagger$ Manager, Advanced Planetary Programs.

§Technical Director, Advanced Planetary Studies. with the solar electric propulsion stage would launch the orbiter and probes in the early 1990s under the current baseline mission scenario. The Saturn probe would be released on approach. An orbiter would be inserted into an initial capture orbit period of 160 days, with subsequent Titan flybys to pump the orbits down to 48 days and, finally, into 32 days, a period having an orbit resonance with Titan of $2 / 1$. This portion of the mission sequence is illustrated in Fig. 1.

Optical navigation is required to obtain the necessary accuracies for these orbital maneuvers; the multiple encounters with Titan further reduce the navigational uncertainties. The Titan probe is released from the 32 day orbit about 10 days prior to encounter with Titan for completion of the probe mission. The orbiter may remain in the 32 day orbit for a series of re-encounters with the probe lander, depending on the class mission being flown. The intermediate probe mission is completed on the initial encounter; the more complex extended surface mission requires several reencounters to complete. Figure 2 illustrates the unusual probe and orbiter encounter geometry with Titan. This is a threebody trajectory geometry since the probe and orbiter are in orbit about Saturn and encounter the path of Titan in such a way that relative to Titan it is retrograde, as illustrated in Fig. 2 relative to Saturn and in Fig. 3 relative to Titan.

Figure 3 illustrates the Titan probe encounter and entry geometry relationship with the orbiter. The probe and orbiter approach Titan in a retrograde trajectory; entry occurs near the subsolar region at about 10:00 a.m. local time. This entry condition was dictated by the science requirement for a lightside entry.

For the encounter and entry sequence, the probe is released about 10 days prior to entry. The orbiter is timed to lag the probe by about $30 \mathrm{deg}$ of longitude at probe entry in order to optimize the communication link geometry during probe descent and surface operation. At an entry radius of $3200 \mathrm{~km}$, the probe enters at a nominal flight path angle of $-30 \mathrm{deg}$ and at a relative velocity of $4.55 \mathrm{~km} / \mathrm{s}$. The orbiter flyby radius of closest approach, when optimized to accommodate the communications link geometry requirements, is a function of the atmospheric model; the closest approach varies 6.1$11.6 R_{\mathrm{T}}$ (Titan radii) for the thin and thick atmospheric models, respectively. The atmospheric models considered in the study, which are defined in the following section, consist of a thin (mostly methane) model and a thick (mostly nitrogen) model. In the thin atmosphere, the probe descends rapidly to the surface; in the thick atmosphere the probe requires an extended period to reach the surface, thus greatly 


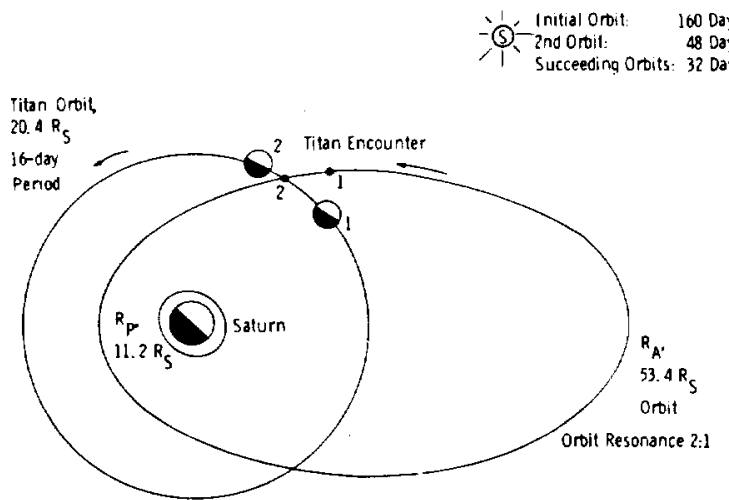

Fig. 1 Encounter geometry, entering Saturn's orbit.

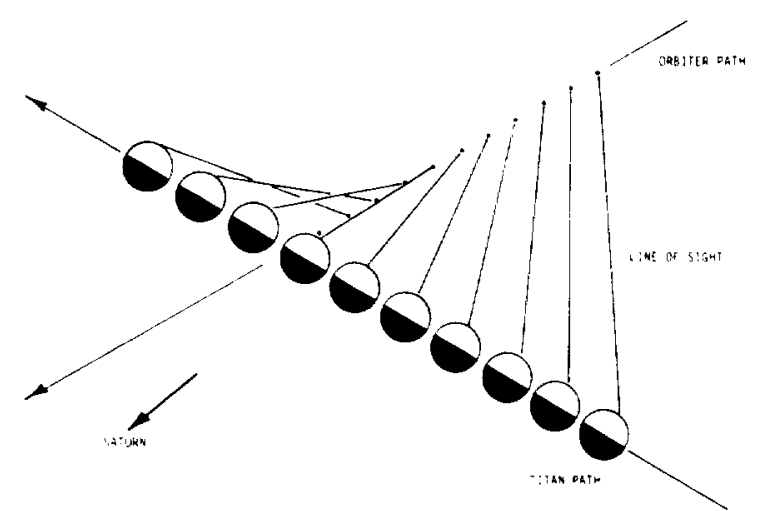

Fig. 2 Encounter geometry at Titan, relative to Saturn.

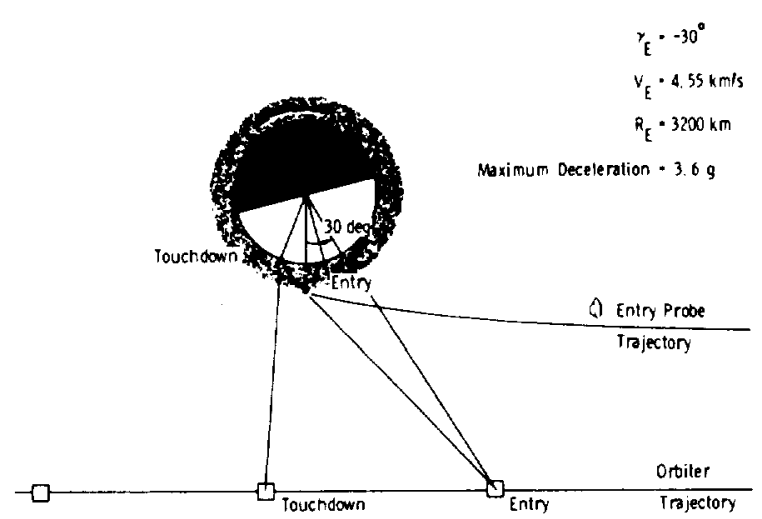

Fig. 3 Entry geometry at Titan, relative to Titan.

affecting the relative communications geometry between the probe and orbiter.

The probe entry and descent sequence is summarized in Fig. 4. The atmospheric model has a significant effect on the mission sequence and timing, as reflected in the descent times. The probe is spin stabilized and oriented in inertial space by the orbiter so that it will have a nearly zero angle of attack during the pre-entry and entry phases. The probe is then released from the Saturn orbiter about 10 days prior to entry. A warmup sequence is begun $5 \mathrm{~h}$ prior to entry to insure that the reference oscillator in the communication subsystem is well stabilized. The pre-entry science instruments are calibrated and pre-entry science data are measured for about $33 \mathrm{~min}$ as the probe descends through the upper atmosphere. At a signal from the accelerometer triad, the pre-entry science module is jettisoned and the probe enters the atmosphere. After entry, when the probe slows to about $0.8 \mathrm{Mach}$ number, a parachute is deployed (in the case of the thin methane atmosphere) to slow the probe further to allow sufficient time for making science measurements and processing the at-

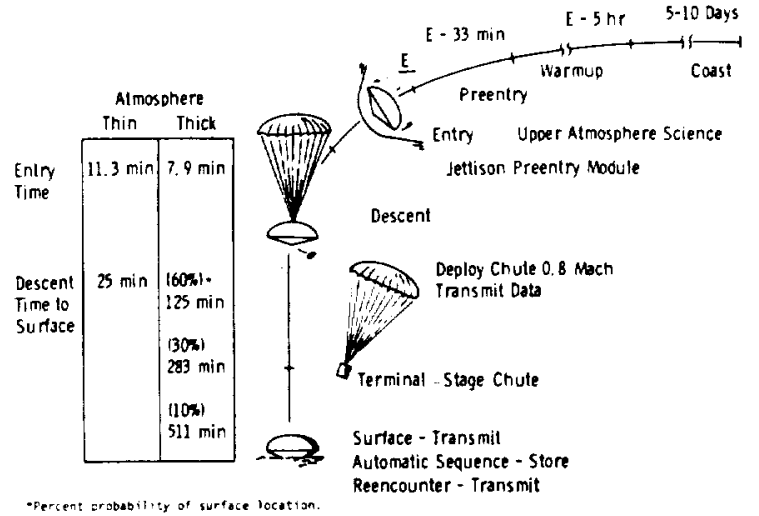

Fig. 4 Mission entry and descent sequence (typical).

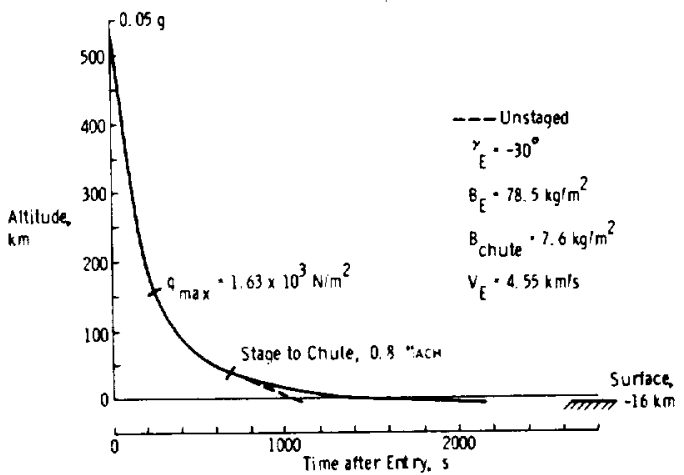

Fig. 5 Entry and descent profile with thin (methane) atmosphere.

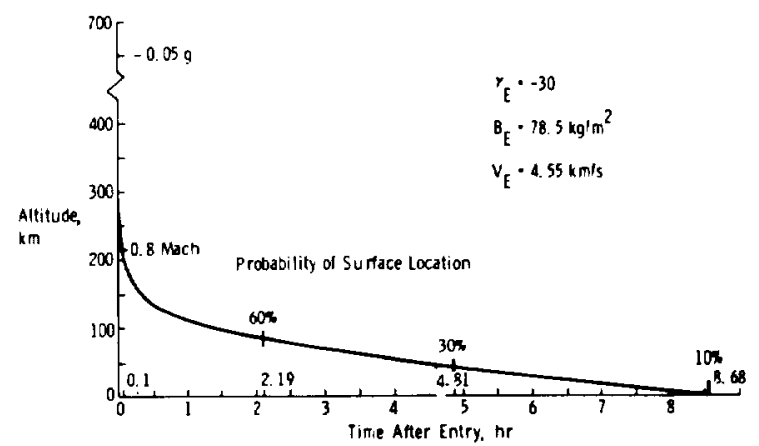

Fig. 6 Entry and descent profile with thick (nitrogen) atmosphere.

mospheric samples. At the time of parachute deployment, the protective covers are jettisoned from the science instruments. At an altitude near the surface, a radar altimeter signals release of the parachute and the probe vehicle free falls to the surface for a hard landing. The probe transmits data to the orbiter during the descent and landing phases of the mission.

The drastic variation in descent times associated with the atmospheric models assumed for the study is shown in Fig. 4. The atmospheric models are unrelated, the thin atmosphere being nearly $100 \%$ methane and the thick atmosphere nearly $100 \%$ nitrogen. The thick atmosphere density and extreme surface location result in a total descent time of about $8.5 \mathrm{~h}$; the descent time in the thin atmosphere is only $7 \mathrm{~min}$ without a parachute and $25 \mathrm{~min}$ with the parachute. Figures 5 and 6 illustrate the effect of the atmospheric model on the descent time history for the thin and thick atmosphere, respectively. In the case of the thin atmosphere, a parachute is deployed after subsonic velocity is reached in order to retard the descent rate further. For the thick atmosphere, the probe retains the entry configuration to the surface.

The surface sequence of Fig. 4 depends on the probe science complement. For the intermediate design discussed in this 
paper, the lander transmits data for $90 \mathrm{~min}$ from the surface only at initial encounter; the more complex lander studied in Ref. 4 also measures and stores science data while the orbiter is out of sight for later transmission at orbiter re-encounter.

\section{Science Requirements}

Titan, the seventh and largest moon of Saturn, is a unique celestial body, certain to yield exciting new phenomena. Titan is sufficiently cold and massive to retain a substantial atmosphere that is composed largely of the reducing gases that are common in the outer solar system. These volatile gases provide interesting possibilities for chemical evolution of organic materials through photochemical production, and their probable fallout to the surface leads to the need for chemical characterization of both the atmosphere and surface of Titan. The benign pre-entry environment allows study of energy sources for the formation of organic compounds and the radiative and dynamic states of the atmosphere, which are intermediate between those of Earth and Venus. This study will provide interesting comparative data. Although current knowledge of Titan's atmosphere is somewhat uncertain, a more accurate atmospheric definition based on new radio astronomy interferometry measurements and on the results of Pioneer Il and Voyager 1 flyby of Titan in 1979 and 1980 , respectively, will be possible before a program start.

For this study both a thin methane-rich atmosphere and a thick nitrogen-rich atmosphere model were included; their characteristics are illustrated in Figs. 7 and 8. The two models are unrelated and represent the possible extremes based on the current state of knowledge about Titan. In the thin methane model the major uncertainty is in surface temperature at the predicted surface radius of $2658 \mathrm{~km}$ and surface pressure of 17 mbars. In the thick nitrogen model, the major uncertainty is assumed to be in the surface temperature and corresponding location denoted in terms of percent-probable surface temperature. This variation results in possible surface pressures of 1.9-21 bars in the thick atmosphere.

In Fig. 8 the triple-point state conditions for each atmosphere are superimposed over the pressure-temperature profiles. In the thin methane atmosphere it is possible to have methane snow or ice at the surface near the areas of lowest temperatures; in the thick nitrogen atmosphere, liquid clouds or even a liquid nitrogen surface are possible near the $60 \%$ probable surface location. This could have a significant effect

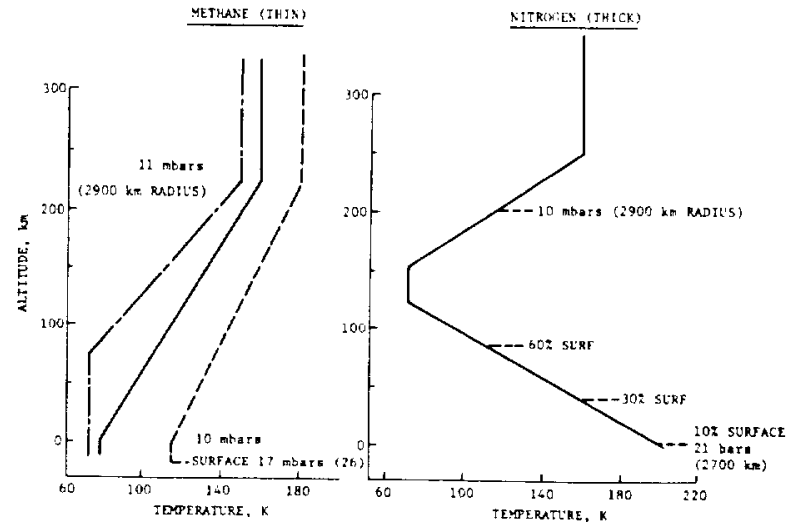

Fig. 7 Titan atmosphere models.
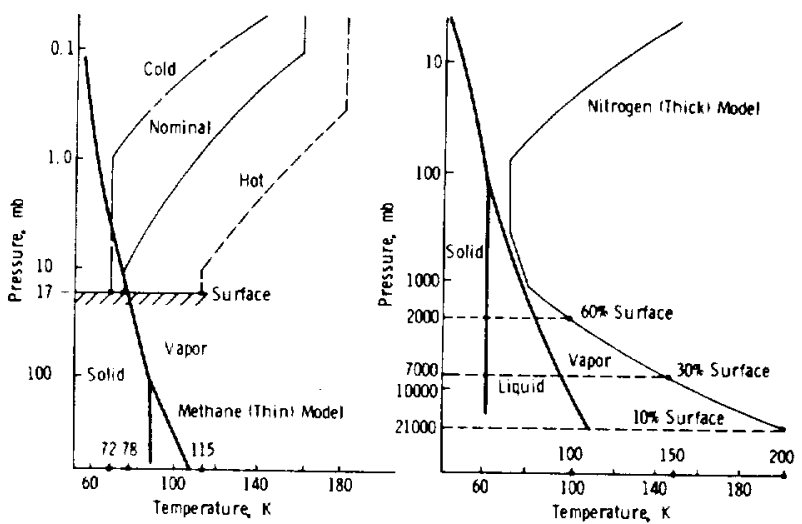

Fig. 8 Titan atmosphere state.

on probe configuration and on surface science sample acquisition.

The science instrument payload characteristics for the intermediate atmosphere and lander probe discussed in this paper are listed in Table 1.

In most of the atmospheric science cases, science instrument definitions were obtained from Galileo probe in. struments. Additional instrument definitions were based on the Pioneer Venus orbiter and probes, on the Viking lander, or on instruments proposed for a Mars penetrator mission.

Table 1 Titan pobe science complement characteristics

\begin{tabular}{|c|c|c|c|}
\hline Instrument & $\begin{array}{c}\text { Weight, } \\
\text { kg }\end{array}$ & $\begin{array}{c}\text { Volume, } \\
\mathrm{cm}^{3}\end{array}$ & $\begin{array}{c}\text { Power, } \\
\text { W }\end{array}$ \\
\hline \multicolumn{4}{|l|}{ Pre-entry } \\
\hline Neutral mass spectrometer (1-46 amu) & 4.3 & 4260 & 11.2 \\
\hline Ion mass spectrometer & 2.9 & 4425 & 1.5 \\
\hline Retarding potential analyzer & 2.7 & 4440 & 2.8 \\
\hline Electron temperature probe & 2.0 & 2050 & 4.0 \\
\hline \multicolumn{4}{|l|}{ Atmosphere } \\
\hline Atmosphere structure instrument & 2.0 & 3100 & 5.5 \\
\hline Multispectral radiometer & 2.5 & 3500 & 4.6 \\
\hline Nephelometer with differential thermal analyzer & 2.5 & 3800 & 2.3 \\
\hline Neutral mass spectrometer (1-150 amu required) & 9.0 & 12,000 & 180 \\
\hline Gas chromatograph & 6.6 & 6500 & 18.0 \\
\hline Descent imagery & 0.25 & 170 & \\
\hline Doppler/wind (orbiter monitors transmitter signal) & 0.0 & & $\begin{array}{l}5.0 / 20 \mathrm{~min} \\
2.0 / 280 \mathrm{~min}\end{array}$ \\
\hline & & & 0.9 \\
\hline \multicolumn{4}{|l|}{ Surface } \\
\hline Impact accelerometer & 0.03 & 30 & 0.03 \\
\hline $\begin{array}{l}\text { Composition (uses atmospheric mass spectrometer, } \\
250 \text {, amu and atmospheric gas chromatograph) }\end{array}$ & & & \\
\hline Meteorology & 0.3 & 300 & 0.075 \\
\hline Surface imaging & 3.0 & 1854 & 4.0 \\
\hline
\end{tabular}


The science instruments are grouped as pre-entry science, atmospheric science, and surface science; they are discussed in the following paragraphs.

\section{Pre-Entry Science}

\section{Objectives}

The objective of the pre-entry science measurements is to characterize the structure, composition, and ionization of the Titan upper atmosphere and ionosphere.

\section{Requirements}

The pre-entry science instruments required to meet the above objective include a neutral mass spectrometer, an ion mass spectrometer, a retarding potential analyzer, and an electron temperature probe. The mass spectrometers are required to measure a mass range of $1-46 \mathrm{amu}$.

The pre-entry science measurements are required from a minimum altitude of about $5000 \mathrm{~km}$ above the surface down to the continuum atmosphere where the entry aerodynamic bow shock forms. For Titan, the entry altitude is about 500 $600 \mathrm{~km}$ above the surface, depending on the atmospheric model assumed.

The pre-entry science instrument data requirements are given in Table 2. The characteristics for these instruments are based on the Pioneer Venus upper atmosphere instruments; the data rate requirements were scaled from previously developed requirements for a Jupiter probe. Differences in approach velocity and flight path angle were taken into account.

The neutral mass spectrometer operates in the ionosphere and upper atmosphere and measures the neutral constituents up to a mass of 46 amu for comparison with the lower atmosphere neutral mass spectrometer data to characterize the total Titan atmosphere.

The ion mass spectrometer operates in the ionosphere to determine the ionic abundances; it provides distribution data as a function of altitude.

The retarding potential analyzer samples the ionic species through the magnetosphere and ionosphere to determine the ion concentrations, ion temperatures, drift velocities, and energy distributions.

The electron temperatures probe (Langmuir probe) obtains electron number densities and temperatures throughout the jonosphere.

\section{Almospheric Science}

Objective

The objective of the atmospheric science measurements is to characterize the atmospheric structure, bulk composition, cloud vertical distribution and composition, and winds.

\section{Requirements}

The atmospheric science instruments required to meet the above objective are: atmosphere structure instrument, multispectral radiometer, nephelometer with differential thermal analyzer, neutral mass spectrometer (1-150 amu), gas chromatograph, Doppler/wind (stable oscillator), and descent imagery (support cloud imagery and surface science). The data requirements and physical descriptions of these instruments are given in Tables 1 and 2.

The atmosphere structure instrument consists of an accelerometer triad and pressure and temperature sensors. Deceleration and pressure data are required throughout the entry and descent phase; temperature data are required during descent.

The multispectral radiometer includes both ultraviolet and visible light channels with a field of view, out and up, of about 90 deg.

The nephelometer measures cloud particle (aerosol) reflectance and density of particles. The differential thermal analyzer (DTA), a small quartz crystal with a resonance frequency that is a very sensitive function of mass loading, provides information on the aerosol composition. It is placed in or parallel to the nephelometer inlet stream, where aerosol is deposited on the crystal. The crystal is heated, and the mass loss at a given temperature is recorded. The unit, which must be deployed into the airstream after the entry heating period is completed, functions during subsonic descent to the surface.

The neutral mass spectrometer, which defines atmospheric and aerosol composition, must cover a mass range of 1-150 amu. Its sample-acquisition device is activated after entry and the spectrometer operates during subsonic descent to the surface.

The gas chromatograph (GC), complements the mass spectrometer (MS) information and also provides composition measurements for atmospheric gases. The current GC designs require considerable time to obtain, process, and purge samples through lengthy columns (about $30 \mathrm{~min}$ for four analyses). It has been estimated that technology ap-

Table 2 Science payload complement data requirements

\begin{tabular}{|c|c|}
\hline Instrument & Data requirements \\
\hline \multicolumn{2}{|l|}{ Pre-entry } \\
\hline Neutral mass spectrometer ( $1-46 \mathrm{amu})$ & $6 \mathrm{bps}$ \\
\hline Jon mass spectrometer & $6 \mathrm{bps}$ \\
\hline Retarding potential analyzer & $18 \mathrm{bps}$ \\
\hline Electron temperature probe & 6 \\
\hline \multicolumn{2}{|l|}{ Atmosphere } \\
\hline Atmosphere structure instrument & $31 \mathrm{bits} / \mathrm{km}$ \\
\hline Multispectral radiometer & $77 \mathrm{bits} / \mathrm{km}$ \\
\hline Nephelometer with differential thermal analyzer & $60 \mathrm{bits} / \mathrm{km}+400 \mathrm{bits} / \mathrm{scale}$ height \\
\hline Neutral mass spectrometer (1-150 amu required) & 25,000 bits total \\
\hline Gas chromatograph & 10,000 bits total \\
\hline Descent imagery & $1.08 \times 10^{6} \mathrm{bits} /$ picture ( 2 minimum) \\
\hline Doppler/wind (stable oscillation) & N/A \\
\hline \multicolumn{2}{|l|}{ Surface } \\
\hline Impact accelerometer & 60,000 bits total \\
\hline Composition, GC/MS (250 amu) & $130,000 \mathrm{bits} / \mathrm{sample}, 10$ samples \\
\hline Meteorology & $0.2 \mathrm{bps}$ \\
\hline Surface imaging & $1.08 \times 10^{6} \mathrm{bits} /$ picture $(2$ minimum) \\
\hline
\end{tabular}


propriate to the Titan mission would allow use of a GC with mass, volume, power, and sample time decreases by a factor of $4 / 10$ over state-of-the-art designs (such as those used in this study).

The descent imagery requires a framing camera (snapshot to stop movement) with sensitive light detection. A CCD (charge-coupled device) imaging camera is appropriate for this application. The estimated surface illumination at the Titan subsolar location is a maximum of about $0.009 \mathrm{~W} / \mathrm{m}^{2}$ and possibly less (by a factor of two) because of clouds. By comparison, Venus surface illumination was measured by the Venera lander at about $40 \mathrm{~W} / \mathrm{m}^{2}$ (a moonlight night on Earth is about $0.003 \mathrm{~W} / \mathrm{m}^{2}$ ). A minimum of two images is required, but more are desired.

For the Doppler/wind-tracking equipment, the telemetry transmitter oscillator on the probe requires a $3 \times 10^{-10} \mathrm{rms}$ phase stability during the descent period. The orbiter receiver must be capable of detecting frequency shifts of this order of magnitude.

\section{Surface Science}

\section{Objective}

The objective of the surface science is to determine the composition and structure of the surface and interior and the properties of organics and precipitants. The surface science also supports the atmospheric science through use of a meteorological experiment that measures local near-surface atmospheric pressure, temperature, and winds.

\section{Requirements}

The surface science instruments required to meet the above objectives are as follows: an impact accelerometer, a mass spectrometer and gas chromatograph for compositional analyses, meteorological instruments, and an instrument for surface imaging. The data requirements and physical descriptions of these instruments are given in Tables 1 and 2.

The impact accelerometer must have a range of $0-3,390$ $\mathrm{m} / \mathrm{s}^{2}(400 \mathrm{~g})$. The hard lander is designed to sustain a maximum deceleration of $2942 \mathrm{~m} / \mathrm{s}^{2}(300 \mathrm{~g})$ on impact with a solid surface. The impact accelerometer stroke time history at impact should provide some information on the surface hardness. The surface may vary in consistency from solid ice to snow to liquid.

The surface composition instrument uses both a mass spectrometer and a gas chromatograpy. The same instruments are also used for atmospheric composition measurements during descent. This experiment requires devices for sample acquisition and sample processing at preselected stepwise temperatures up to $200-300 \mathrm{~K}$ in a sealed oven so that gaseous products can be carried into the detecting instruments. In addition, suitable manifolding is required to deliver the gaseous samples either to the $\mathrm{GC}$ and $\mathrm{MS}$ in parallel (separately) or in series first through the GC and then into the MS. The MS must have a mass range of 1-250 amu in order to cover the anticipated range of surface materials (which may include ices, clathrates, and organic materials).

The meteorological experiment includes sensors for atmospheric pressure, temperature, and wind velocity and direction. The wind detector and temperature sensors should be mounted at least $1 \mathrm{~m}$ above the surface. The descent pressure sensor is also used for this experiment.

The surface imaging requires a high-quality color image (three black-and-white images with appropriate filters). The imager may be a CCD-type or a facsimile-type camera. With a CCD, the analog data are read directly into an analog delay line buff device just as in the descent imaging equipment; the data are then digitized and transmitted to the orbiter. The facsimile-type camera is scanned at such a rate that the data are transmitted directly to the orbiter as they are generated and, therefore, a large-scale buffer or data storage is not required. With appropriate filters, both color data and in- frared spectral information are obtained from the sensors. With a 12 bit word per pixel and 3 images per color picture, a data requirement of about $4 \times 10^{6}$ bits per color picture results.

Other surface science instruments considered in the basic study of Ref. 1 included a passive seismometer, a microscope, a precipitation experiment, and an alpha-backscatter composition instrument. Most of these instruments require more than $90 \mathrm{~min}$ to collect a significant data sample.

\section{Probe Design}

The probe configuration was selected to meet the requirements imposed by both atmospheric and surface constraints and still provide a relatively low-cost, uncomplicated design. The probe is required to obtain pre-entry, entry, descent, and surface science measurements and to relay these data to the Saturn orbiter during the initial encounter. The two atmospheric models were considered and probe concepts were designed for each case. These two separate atmospheric models consist of a thin methane model with surface pressures of about 17 mbars and a thick nitrogen model with surface pressures varying 1.9-21 bars. Probe systems are designed for only the thin or thick atmosphere but not for both simultaneously. This approach was based on the assumption that the atmosphere will be more accurately defined before the actual hardware design phase starts. With the currrent models of Titan it is possible that the surface condition may vary from solid ice and snow to liquid; this possibility significantly affects the lander configuration as well as surface sample acquisition.

A broad range of configuration options was considered, as illustrated in Fig. 9, and various concepts were sought that could meet the system design constraints. The configuration design drivers are 1) controlled descent time to provide sufficient time for science measurement and data transmission, and 2) controlled landing to provide low enough impact velocity and shock attenuation to insure a safe, stable landing. As illustrated in the upper right side of Fig. 9, the thin atmosphere required that the lander be retarded by a parachute to allow sufficient time for the descent science measurements (the gas chromatograph requires about 25-30 min to obtain four samples). In a thick atmosphere, the entry probe is slowed to such an extent that its configuration either has to be streamlined to increase its ballistic coefficient or some weight penalties have to be tolerated to accommodate the necessary increased battery life and extended data transmission ranges. Evaluation of this tradeoff resulted in retaining the entry aeroshell on the surface of the probe. Although this configuration had an extended descent time, it resulted in a much simpler landing system. The streamlined shapes have poor landing stability and require the addition of erection devices after touchdown. Part of this tradeoff study included an evaluation of hard vs soft lander concepts, as illustrated in Fig. 9.

The soft lander concept, similar to the Viking lander, can provide a more precise touchdown velocity at the expense of additional system complexity. Guidance and control, attitude control, and terminal propulsion subsystems are necessary, as well as landing legs to provide impact attenuation and attitude stability on the surface.

The hard lander, which is a less complex concept, uses a crushable honeycomb structure behind the entry heat shield for impact attenuation. It is designed to provide less than 300 $g$ deceleration on a hard surface with a stroke of $15 \mathrm{~cm}$ at a descent rate of $20 \mathrm{~m} / \mathrm{s}$. After further study, it became apparent that the hard lander concept provides a practical Titan design: the exploration probe has good impact stability, low penetration on ice or snow, and provides flotation in case of landing on a liquid nitrogen surface. Experience with drop tests of similar shapes in the desert [ with the planetary atmospheric entry test (PAET) vehicle] showed very stable and nonrebounding landings. 


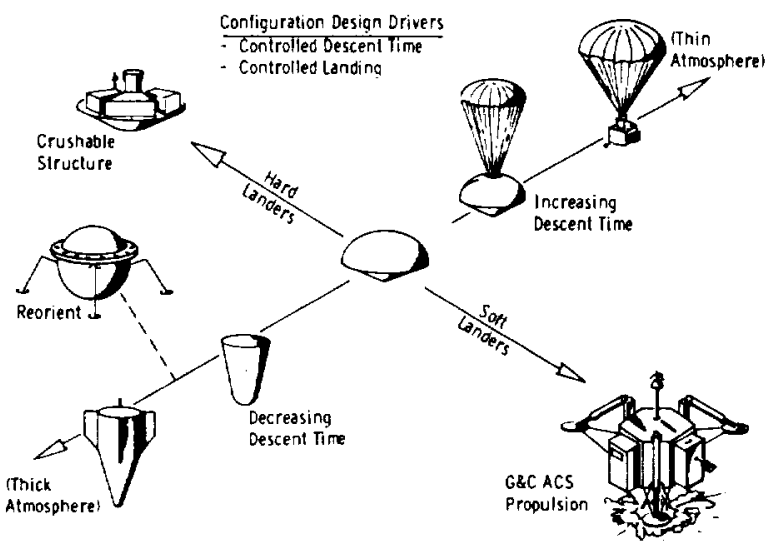

Fig. 9 Configuration options considered.

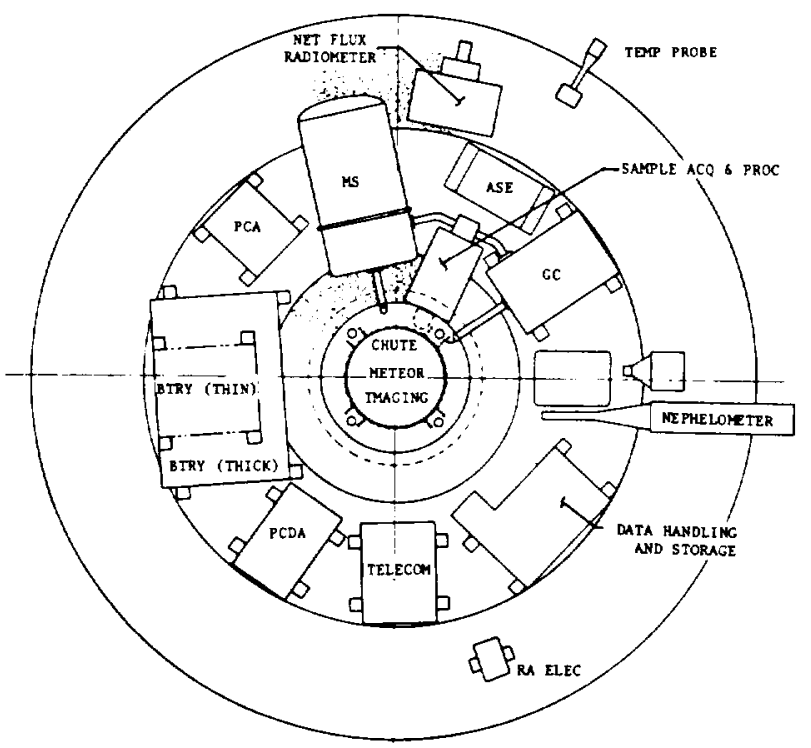

Fig. 10 Class B probe and lander configuration.

The probe concept for the thin methane atmosphere has been designed for the worst-case uncertainties within the thin atmosphere definition. In the case of a thick nitrogen atmosphere, the baseline probes have been designed to meet the $60 \%$ probable and $30 \%$ probable surface conditions but not the $10 \%$ probable surface conditions. It was felt that the $10 \%$ probable surface condition requirement would lead to unrealistic probe designs. However, the effect of this extreme was evaluated as a tradeoff to the baseline design and the results showed that the system mass increased by $30 \%$.

The external configuration, detailed in Figs. 10 and 11 , used a $1.47 \mathrm{~m}$ diam, $70 \mathrm{deg}$ half-angle cone aeroshell and ablator with a segment of a sphere for the aft cover. The combination aeroshell and landing-impact attenuator incorporate a honeycomb structure that limits the impact deceleration to less than $300 \mathrm{~g}$ with a $15 \mathrm{~cm}$ stroke of the crushable honeycomb at an impact velocity of $20 \mathrm{~m} / \mathrm{s}$. This approach simplifies the landing system considerably compared with that of a Viking-type soft lander, since neither the aeroshell nor the aft cover has to be staged and there is no requriement for terminal guidance or propulsion.

The configuration shown was designed for the thin (methane) atmosphere and, therefore, requires a parachute to slow descent rate. The parachute is mounted at the top of a central structural tube which encloses the extendible science mast. This mast, used to deploy the meteorological and imaging experiments after touchdown, carries a second microstrip-type if antenna on top because the descent antenna

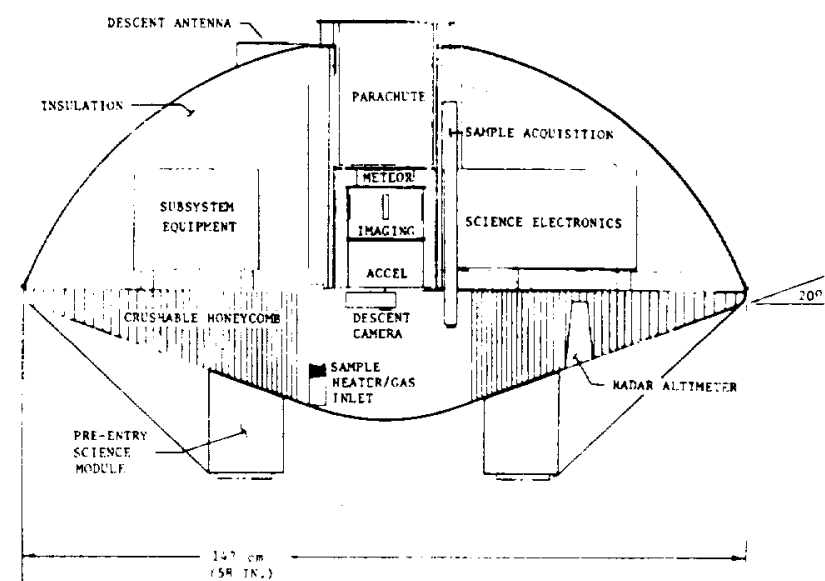

Fig. 11 Class B probe and lander (plan view).

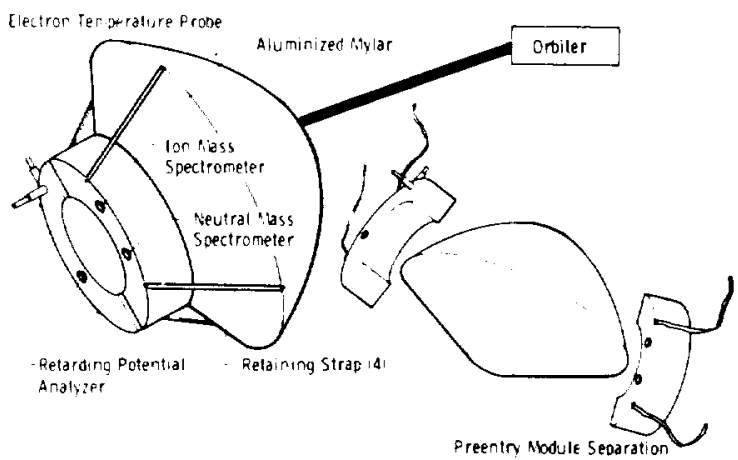

Fig. 12 Pre-entry science module.

may be blocked from the line of sight to the orbiter after touchdown.

This probe lander design features a jettisonable nose cap with a cylindrical cutout in the center of the vehicle to provide access to science samples both during atmospheric descent and on the surface. The nose-cap separation takes place just after entry about $0.8 \mathrm{Mach}$ number.

A collapsible atmospheric sampling inlet provides sample gases to the mass spectrometer and gas chromatograph during descent; it collapses at touchdown. A sample acquisition drill device is then deployed to the surface to obtain surface samples.

A $7.0 \mathrm{~cm}$ thick layer of insulation (density of $64 \mathrm{~kg} / \mathrm{m}^{3}$ ) attached to the back cover encloses the components. The components are mounted on thermal isolation standoffs that are attached to the honeycomb back plate with insulation between the two. The honeycomb structure provides load paths from the components to the aeroshell without ad-

Table 3 Probe mass breakdown

\begin{tabular}{lcc}
\hline & \multicolumn{2}{c}{ Probe mass, kg } \\
\cline { 2 - 3 } & $\begin{array}{c}\text { Thin } \\
\text { atmosphere }\end{array}$ & $\begin{array}{c}\text { Thick } \\
\text { atmosphere }\end{array}$ \\
\hline Science plus margin & 51.0 & 51.0 \\
Telecommunications & 2.6 & 2.6 \\
Power/pyro/cabling & 29.4 & 43.6 \\
Thermal & 21.0 & 16.4 \\
Structures/mechanisms & & \\
parachute & 81.5 & 69.5 \\
Data handling and control & 12.56 & 12.56 \\
$\quad$ Subtotal & 197.3 & 196.47 \\
15\% contingency & 29.6 & 29.47 \\
$\quad$ Total & 226.9 & 225.94 \\
\hline \hline
\end{tabular}

${ }^{3} 30 \%$ probable surface location. 
ditional frames. The parachute loads are carried through the cylindrical mast enclosure tube down into the honeycomb backing plate.

In this hard lander concept the relatively flat aeroshell provides good impact and attitude stability on the surface and prevents surface penetration; the configuration will float if it lands on a liquid nitrogen surface. The aft cover thermally insulates the components and protects them from possible splash or debris at touchdown.

For the thin-atmosphere probe design, an $8 \mathrm{~m}$ diam parachute is required to control the touchdown descent velocity to within $20 \mathrm{~m} / \mathrm{s}$ and a resulting maximum impact deceleration of $300 \mathrm{~g}$ with the $15 \mathrm{~cm}$ attenuator stroke. In the proposed concept, the parachute is designed to provide a velocity at an altitude of $100 \mathrm{~m}$ that results in a $20 \mathrm{~m} / \mathrm{s}$ touchdown velocity after free fall from $100 \mathrm{~m}$. The parachute can be rigged to drift to the side of the lander and, by retaining the canister on the risers, the parachute will remain inflated. A small radar altimeter is used to signal the $100 \mathrm{~m}$ release altitude; it is located in the honeycomb area as illustrated in Fig. 11. An rf-transparent cover provides line of sight through the aeroshell.

The pre-entry science module is illustrated in Fig. 12. The unit is strapped onto the forward heat shield with straps that are attached by pins in notches at the maximum diameter of the vehicle. The module is separated into two parts when the redundant hot-wire tension links are severed. At that point, three forces provide positive separation of the module from the entry vehicle. These forces are produced by the springs between the module halves, the centrifugal force due to the spinning vehicle and any low-level aerodynamic loads that may be building up at time of release. The science instruments are located as shown to insure clean, uncontaminated samples of the upper atmosphere.

The total probe mass is summarized in Table 3 by subsystem grouping. The mass of the probe designed for the thin (methane) atmosphere is $226.9 \mathrm{~kg}$, and that of the thickatmosphere probe $225.94 \mathrm{~kg}$. Although the total masses are nearly identical, the subsystem masses vary considerably. The basic difference is that the thin-atmosphere probe requires a parachute and the thick atmosphere probe requires additional batteries to accommodate the longer descent time.

\section{Subsystem Design}

The science sequences and resultant data rate requirements are based on science instrument measurement periods and sampling intervals. Both the pre-entry and entry science data are stored for later transmission during the subsonic descent phase. The rapid descent in the thin methane atmosphere results in relatively high data rates of about 1500 bps compared to 750 bps in the thick nitrogen atmosphere. Once on the surface, the data rates increase to about 2300 bps to accommodate the large volume of data generated by the surface science and imaging camera. These data must be transmitted before the orbiter travels out of range or over the horizon.

Because of differences in the communication link geometry, the thin-atmosphere probe design requires a $2.5 \mathrm{~W}$ rf output transmitter; the thick-atmosphere design, with its greater range, requires a $6.0 \mathrm{~W}$ transmitter. The greater probe-to-orbiter range of the latter case results from the increased orbiter flyby altitude required to provide the extended time of $8.5 \mathrm{~h}$ involved in descent in the thick atmosphere.

The data-handling system requires a buffer memory capacity of $135 \mathrm{~K}$ bits to handle the pre-entry and entry data storage. For the CCD-type imaging camera used during descent, the data are buffered in an analog delay-line device in analog format for later conversion to a digital data stream prior to transmission.

Because of the $7-8$ yr flight time prior to probe release, a silver-zinc, remotely activated primary battery power system was selected. This provides a proved concept with the necessary reliability for this application. The energy required for the thin-atmosphere design is $260 \mathrm{~W} \cdot \mathrm{h}$ compared to 800 $\mathrm{W} \cdot \mathrm{h}$ for the thick-atmosphere design.

The thermal control system uses isotope heaters and electrical equipment waste heat in conjunction with insulation to maintain internal temperature within limits. During the pre-entry coast phase, excess heat is dumped overboard through use of heat pipes and a base cover radiator. During descent, the atmosphere is extremely cold and conduction loss requires use of all the internal heat and isotope heater energy available to hold the internal temperature within limits.

\section{Conclusions}

The technical requirements, conceptual design, and science return of probes for use in Titan exploration have been reviewed. Based on study results, the following conclusions are made:

1) The Titan exploration probe concept is feasible in both thick and thin atmospheres. The thin-atmosphere design requires a parachute to provide sufficient descent time for science, and the thick-atmosphere design requires additional battery power to accommodate lengthy descent times.

2) The hard lander concept is a practical design approach for either atmospheric model. The hard lander configurations provide good impact stability, low penetration on ice or snow, and flotation on liquid surface.

3) The probe designs meet the overall science requirements without major new developments.

4) A probe design that meets the mission requirements for the exploration of Titan has a mass of about $227 \mathrm{~kg}$ with a 1.5 $m$ diam entry aeroshell.

\section{References}

1 "The Saturn System," NASA CP.2068, 1978.

${ }^{2}$ Wallace, R.A., "1977 Saturn Mission Options Study," Jet Propulsion Laboratory, Rept. 710-6, Sept. 1977.

${ }^{3}$ Wright, J.L., "Saturn Orbiter Dual Probe Mission Concept: A Preliminary Description," Jet Propulsion Laboratory, Rept. 710-20, Sept. 1978.

4" SStudy of Entry and Landing Probes for Exploration of Titan," NASA CR-152275, 1979. 